\title{
Growth and nutritional status, and their association with lung function: a study from the international Primary Ciliary Dyskinesia Cohort
}

Myrofora Goutaki (1) ${ }^{1,2}$, Florian S. Halbeisen ${ }^{1}$, Ben D. Spycher ${ }^{1}$ Elisabeth Maurer ${ }^{1}$, Fabiën Belle ${ }^{1}$, Israel Amirav $\mathbb{1}^{3,4}$ on behalf of the PCD Israeli Consortium, Laura Behan ${ }^{5,6}$, Mieke Boon ${ }^{7}$, Siobhan Carr ${ }^{8}$, Carmen Casaulta $^{2}$ on behalf of the Swiss PCD Group, Annick Clement ${ }^{9}$ on behalf of the French Reference Centre for Rare Lung Diseases, Suzanne Crowley ${ }^{10}$, Sharon Dell ${ }^{11}$, Thomas Ferkol ${ }^{12}$, Eric G. Haarman ${ }^{13}$, Bulent Karadag ${ }^{14}$, Michael Knowles ${ }^{15}$, Cordula Koerner-Rettberg ${ }^{16}$, Margaret W. Leigh ${ }^{17}$, Michael R. Loebinger ${ }^{18}$, Henryk Mazurek ${ }^{19}$, Lucy Morgan ${ }^{20}$, Kim G. Nielsen ${ }^{21}$, Maria Phillipsen ${ }^{21}$, Scott D. Sagel ${ }^{22}$, Francesca Santamaria ${ }^{23}$, Nicolaus Schwerk ${ }^{24}$, Panayiotis Yiallouros ${ }^{25}$, Jane S. Lucas $\mathbb{1}^{5}$ and Claudia E. Kuehni (1) ${ }^{1}$

@ERSpublications

Multidisciplinary management and nutritional advice could improve growth and delay lung function impairment in PCD http://ow.ly/5iQz30gB4Mo

Cite this article as: Goutaki M, Halbeisen FS, Spycher BD, et al. Growth and nutritional status, and their association with lung function: a study from the international Primary Ciliary Dyskinesia Cohort. Eur Respir J 2017; 50: 1701659 [https://doi.org/10.1183/13993003.01659-2017].

ABSTRACT Chronic respiratory disease can affect growth and nutrition, which can influence lung function. We investigated height, body mass index (BMI), and lung function in patients with primary ciliary dyskinesia (PCD).

In this study, based on the international PCD (iPCD) Cohort, we calculated z-scores for height and BMI using World Health Organization (WHO) and national growth references, and assessed associations with age, sex, country, diagnostic certainty, age at diagnosis, organ laterality and lung function in multilevel regression models that accounted for repeated measurements.

We analysed 6402 measurements from 1609 iPCD Cohort patients. Height was reduced compared to WHO (z-score $-0.12,95 \% \mathrm{CI}-0.17$ to -0.06 ) and national references ( $\mathrm{z}$-score $-0.27,95 \% \mathrm{CI}-0.33$ to $-0.21)$ in male and female patients in all age groups, with variation between countries. Height and BMI were higher in patients diagnosed earlier in life ( $p=0.026$ and $p<0.001$, respectively) and closely associated with forced expiratory volume in $1 \mathrm{~s}$ and forced vital capacity $\mathrm{z}$-scores $(\mathrm{p}<0.001)$.

Our study indicates that both growth and nutrition are affected adversely in PCD patients from early life and are both strongly associated with lung function. If supported by longitudinal studies, these findings suggest that early diagnosis with multidisciplinary management and nutritional advice could improve growth and delay disease progression and lung function impairment in PCD. 
Affiliations: ${ }^{1}$ Institute of Social and Preventive Medicine, University of Bern, Bern, Switzerland. ${ }^{2}$ Paediatric Respiratory Medicine, Children's University Hospital of Bern, University of Bern, Bern, Switzerland. ${ }^{3}$ Dept of Pediatrics, Faculty of Medicine, Bar Ilan University, Ramat Gan, Israel. ${ }^{4}$ Dept of Pediatrics, Faculty of Medicine and Dentistry, University of Alberta, Edmonton, Canada. ${ }^{5}$ Primary Ciliary Dyskinesia Centre, NIHR Respiratory Biomedical Research Centre, University of Southampton, Southampton, UK. 'School of Applied Psychology, University College Cork, Cork, Ireland. ${ }^{7}$ Dept of Paediatrics, University Hospital Gasthuisberg, Leuven, Belgium. ${ }^{8}$ Primary Ciliary Dyskinesia Centre, Dept of Paediatrics, Royal Brompton and Harefield Foundation Trust, London, UK. ${ }^{9}$ Paediatric Pulmonary Dept, Trousseau Hospital APHP, Sorbonne Universities and Pierre et Marie Curie University, Paris, France. ${ }^{10}$ Unit for Paediatric Heart, Lung, Allergic Diseases, Rikshospitalet, Oslo, Norway. ${ }^{11}$ Dept of Pediatrics, SickKids Hospital, University of Toronto, Toronto, Canada. ${ }^{12}$ Dept of Pediatrics, School of Medicine, Washington University, St Louis, MO, USA. ${ }^{13}$ Dept of Pediatric Pulmonology, VU University Medical Center, Amsterdam, The Netherlands. ${ }^{14}$ Dept of Pediatric Pulmonology, School of Medicine, Marmara University, Istanbul, Turkey. ${ }^{15}$ Dept of Medicine, Marsico Lung Institute, School of Medicine, University of North Carolina, Chapel Hill, NC, USA. ${ }^{16}$ Dept of Paediatric Pneumology, University Children's Hospital, Ruhr University, Bochum, Germany. ${ }^{17}$ Dept of Pediatrics, Marsico Lung Institute, School of Medicine, University of North Carolina, Chapel Hill, NC, USA. ${ }^{18}$ Host Defence Unit, Royal Brompton and Harefield NHS Foundation Trust, London, UK. ${ }^{19}$ Dept of Pneumonology and Cystic Fibrosis, Institute of Tuberculosis and Lung Disorders, Rabka-Zdrój, Poland. ${ }^{20}$ Dept of Respiratory Medicine, Concord Hospital Clinical School, University of Sydney, Sydney, Australia. ${ }^{21}$ Danish PCD Centre Copenhagen, Paediatric Pulmonary Service, Copenhagen University Hospital, Copenhagen, Denmark. ${ }^{22}$ Dept of Pediatrics, University of Colorado School of Medicine, Aurora, CO, USA. ${ }^{23}$ Dept of Translational Medical Sciences, Federico II University, Napoli, Italy. ${ }^{24} \mathrm{Clinic}$ for Paediatric Pulmonology, Allergiology and Neonatology, Hannover Medical School, Hannover, Germany. ${ }^{25}$ School of Medicine, University of Cyprus, Nicosia, Cyprus.

Correspondence: C.E. Kuehni, Institute of Social and Preventive Medicine, University of Bern, Finkenhubelweg 11, Bern 3012, Switzerland. Email: claudia.kuehnidispm.unibe.ch

\section{Introduction}

Chronic respiratory disease could delay growth and weight gain in children, and lead to reduced height and body mass index (BMI) in adults. Both disease and its respective treatments could cause this delay $[1,2]$. Children with asthma tend to grow more slowly because of higher resting energy expenditure [3] and delayed pubertal onset [4], or corticosteroid medication [5]. Growth and nutrition in childhood are also associated with lung function later in life, in patients with cystic fibrosis (CF) and bronchopulmonary dysplasia [6,7]. Monitoring BMI and height is thus part of state-of-the art care of patients with chronic lung diseases, such as chronic obstructive pulmonary disease and CF $[8,9]$, just as they are used as indicators of growth and nutritional status in healthy individuals [10]. Though growth and nutrition in children with common lung diseases like asthma are well studied, we still know little about height and BMI in patients with rare pulmonary diseases, such as primary ciliary dyskinesia (PCD).

The genetically heterogeneous disease PCD affects approximately one in 10000 people; though rare, it is likely underdiagnosed [11]. It is characterised by mutations that impair the function of motile cilia, thereby affecting organs throughout the body, the respiratory system in particular [12]. The few studies published on growth so far, have been contradictory; some suggest impaired growth [13-15], while others do not $[16,17]$. All previous studies were relatively small, included mainly children, and came from one or only a few centres. A further limitation of existing research originates in the reference values for height and BMI. These values vary between countries, yet studies have rarely used national reference values [15, 17], which are necessary if the growth of PCD patients is to be compared with that of their healthy peers.

We hypothesised that the height and BMI of PCD patients are already impaired in early childhood, and are associated with lung function, as they are in other severe lung diseases. We obtained the height and BMI values of patients in the international PCD (iPCD) Cohort, which we compared to national and

Support statement: The development of the international Primary Ciliary Dyskinesia (iPCD) Cohort is funded by the European Union's Seventh Framework Programme (EG-GA No.35404 BESTCILIA: Better Experimental Screening and Treatment for Primary Ciliary Dyskinesia). The primary ciliary dyskinesia (PCD) research at the Institute of Social and Preventive Medicine (ISPM) in Bern is supported by the Swiss National Science Foundation (SNF 320030_173044) and receives national funding from the Lung Leagues of Bern, St. Gallen, Vaud, Ticino and Valais, as well as the Milena-Carvajal Pro Kartagener Foundation. C. Kuehni is supported by the Swiss National Science Foundation (SNF32003B_162820 and SNF32003B_144068) while the national PCD service in Southampton and London is funded by the National Health Service (NHS) in England. The researchers participate in the Better Evidence to Advance Therapeutic Options for PCD (BEAT-PCD) network (COST action BM 1407). A Swiss National Science Foundation fellowship (PZ00P3_147987) supports B.D. Spycher. Swiss Cancer Research grants (KLS-3412-02-2014 and KLS-3644-02-2015) support F. Belle. The AAIR Charity (Reg. No. 1129698) supports L. Behan. S. Dell, T. Ferkol, M. Knowles, M.W. Leigh and S.D. Sagel are supported by the National Institutes of Health (U54HL096458). S.D. Sagel is also supported by local funding (CTSA NIH/NCATS Colorado UL1TR000154). Funding information for this article has been deposited with the Crossref Funder Registry.

Conflict of interest: Disclosures can be found alongside this article at erj.ersjournals.com 


\begin{tabular}{|c|c|}
\hline Characteristic & $\mathrm{n}(\%)^{\#}$ \\
\hline \multicolumn{2}{|l|}{ Sex } \\
\hline Male & 780 (49) \\
\hline Female & $829(51)$ \\
\hline \multicolumn{2}{|l|}{ Country of residence ${ }^{\Uparrow}$} \\
\hline Australia & $55(3)$ \\
\hline Northern Europe & $390(24)$ \\
\hline Western Europe & $405(25)$ \\
\hline Eastern Europe & $97(6)$ \\
\hline Southern Europe & $46(3)$ \\
\hline Western Asia & 202 (13) \\
\hline North America & $414(26)$ \\
\hline \multicolumn{2}{|l|}{ Organ laterality } \\
\hline Situs solitus & $827(51)$ \\
\hline Situs inversus & 587 (37) \\
\hline Heterotaxia & $51(3)$ \\
\hline Situs status not reported & $144(9)$ \\
\hline \multicolumn{2}{|l|}{ Date of birth } \\
\hline Earlier than 1976 & $252(16)$ \\
\hline $1977-1996$ & $497(31)$ \\
\hline $1997-2015$ & 860 (53) \\
\hline \multicolumn{2}{|l|}{ Diagnostic information } \\
\hline Definite PCD diagnosis ${ }^{+}$ & $1056(66)$ \\
\hline Probable PCD diagnosis ${ }^{\S}$ & $256(16)$ \\
\hline Clinical diagnosis only & 297 (18) \\
\hline \multicolumn{2}{|l|}{ Age at diagnosis years } \\
\hline $0-9$ & $800(50)$ \\
\hline $10-19$ & $451(28)$ \\
\hline $20-29$ & $129(8)$ \\
\hline $30-39$ & $93(6)$ \\
\hline $40-49$ & $72(4)$ \\
\hline$\geqslant 50$ & $64(4)$ \\
\hline
\end{tabular}

\#: Total number of subjects (N) was 1609. ๆ: Northern Europe: Denmark, Norway, United Kingdom; Western Europe: Belgium, France, Germany, Switzerland, The Netherlands; Eastern Europe: Poland; Southern Europe: Italy, Serbia; Western Asia: Cyprus, Israel, Turkey; North America: Canada, United States (based on the geographical region definitions of the United Nations Statistics Division. August 2016). ${ }^{+}$: defined as hallmark primary ciliary dyskinesia (PCD) electron microscopy findings and/or biallelic gene mutation, identified based on the ERS PCD Diagnostics Task Force guidelines. ${ }^{\S}$ : abnormal light or high frequency video microscopy finding and/or low $\left(\leqslant 77 \mathrm{~nL} \cdot \mathrm{min}^{-1}\right.$ ) nasal NO value.

international reference values. We investigated determinants of poor growth, including sex, age, country of residence and level of diagnostic certainty. We also investigated growth in paediatric patients and its dependence on the age when the patients were diagnosed with PCD, and whether their growth was associated with lung function.

\section{Methods}

Study population and study design

The iPCD Cohort is a large, retrospective international cohort of PCD patients developed during the EU FP7 (Seventh Framework Programme) project Better Experimental Screening and Treatment for Primary Ciliary Dyskinesia (BESTCILIA). It includes demographic data and information on diagnostic tests, clinical symptoms, growth, lung function and treatment. Clinical and diagnostic data from over 3000 PCD patients in 18 countries allow researchers to pose questions about clinical phenotype, natural history, prognostic factors and effect of treatments. Details of the iPCD Cohort have been described elsewhere [18]. For the present study, we included all iPCD datasets that had been delivered, cleaned and standardised by the time of the analysis (April 2016), and had cross-sectional information on height and weight. Information on ethical approval can be found online in the supplementary material.

\section{PCD diagnosis}

The PCD diagnostics now available have evolved rapidly [19]. Current recommendations include a combination of tests [20], but test availability differs between countries [19]. Thus, PCD patients have not 
been diagnosed in similar ways. We divided patients in the iPCD Cohort, who all had a strong clinical suspicion, into three diagnostic subgroups, based on the results of available tests. The first subgroup included patients with definite PCD, which was defined, based on recent guidelines of the ERS PCD Diagnostics Task Force [20], by hallmark transmission electron microscopy findings and/or an identified biallelic PCD genetic mutation. The second subgroup, probable PCD, included patients with abnormal high-speed video microscopy findings and/or low nasal nitric oxide (nNO). The third subgroup included patients with a clinical PCD diagnosis. The supplementary material describes the diagnostic details.

\section{Height and BMI}

We checked data quality to identify outliers and implausible values, and contacted contributors when necessary, to resolve any such matters. We calculated age- and sex-adjusted height and BMI z-scores, based on international reference values from the World Health Organisation (WHO) [21] and national reference values (sources are listed in supplementary table S1).

Growth references available for height and BMI were intended for persons up to 19 years of age (or in some countries, up to the age of 20). For patients aged $<20$ years, we calculated height and BMI z-scores, based on the exact age-specific references. For patients aged $\geqslant 20$ years, we calculated height $z$-scores, based on the reference values for 19-year-olds; these describe final adult height. We also calculated BMI z-scores for adults, based on the reference values for 19-year-olds, because no BMI z-score references presently exist for adults. We defined short stature as a height $\mathrm{z}$-score $\leqslant-2$; underweight, as a BMI $\mathrm{z}$-score $\leqslant-2$; and overweight, as a BMI $\mathrm{z}$-score $\geqslant 2$, according to the definitions used by WHO.

\section{Determinants of height and BMI}

We investigated associations with height and BMI among the following factors: sex, age, country of residence, level of diagnostic certainty, age at diagnosis, organ laterality and lung function at time of height measurement. Growth might differ between male and female patients with PCD, which is manifested as slow growth and/or malnutrition in childhood, and decreased final adult height. Differences between countries could reveal ethnic variations or differences in disease management. In children, diagnosis at a younger age might improve growth, as it would allow an earlier introduction of optimal disease management. Patients with situs inversus might have less severe disease, because they are generally diagnosed earlier, before symptoms develop. Details regarding how we categorised the variables chosen are in the supplementary material.

\section{Lung function}

We chose forced expiratory volume in $1 \mathrm{~s}$ (FEV1), and forced vital capacity (FVC) as indicators of lung function, and used the Global Lung Function Initiative (GLI) reference values to calculate age, sex, ethnicity and height-adjusted z-scores [22]. All lung function measurements were checked for quality, and since 2005, they have been performed according to the European Respiratory Society/American Thoracic

\begin{tabular}{|c|c|c|c|c|c|}
\hline Characteristic & Population & Reference & Subjects & z-score & p-value \\
\hline \multirow[t]{6}{*}{ Height } & Overall study & National ${ }^{\#}$ & 1601 & $-0.27(-0.33$ to -0.21$)$ & $<0.001$ \\
\hline & Overall study & WHO & 1609 & $-0.12(-0.17$ to -0.06$)$ & $<0.001$ \\
\hline & Paediatric $(<20$ years) & National $^{\#}$ & 1226 & $-0.26(-0.33$ to -0.20$)$ & $<0.001$ \\
\hline & Adult ( $\geqslant 20$ years) & National $^{\#}$ & 439 & $-0.31(-0.42$ to -0.20$)$ & $<0.001$ \\
\hline & Definite PCD & National $^{\#}$ & 1054 & $-0.26(-0.33$ to -0.19$)$ & $<0.001$ \\
\hline & Overall study $^{+}$ & National ${ }^{\#}$ & 1601 & $-0.30(-0.36$ to -0.24$)$ & $<0.001$ \\
\hline \multirow[t]{5}{*}{ BMI } & Overall study & National ${ }^{\#}$ & 1549 & $0.06(0.002$ to 0.13$)$ & 0.043 \\
\hline & Overall study & WHO & 1539 & 0.21 (0.14 to 0.27$)$ & $<0.001$ \\
\hline & Paediatric $(<20$ years) & National ${ }^{\#}$ & 1184 & $0.02(-0.05$ to 0.09$)$ & 0.582 \\
\hline & Definite PCD & National ${ }^{\#}$ & 1019 & $0.05(-0.02$ to 0.13$)$ & 0.172 \\
\hline & Overall study ${ }^{+}$ & National $^{\#}$ & 1549 & $0.03(-0.04$ to 0.09$)$ & 0.424 \\
\hline
\end{tabular}

Data is presented as $\mathrm{n}$ or mean $(95 \% \mathrm{Cl})$ unless otherwise stated. \# : patients from Serbia were excluded from this analysis because no national references were available. ๆ: defined as hallmark PCD electron microscopy findings and/or biallelic gene mutation identified based on the ERS PCD Diagnostics Task Force guidelines. ${ }^{+}$: analysis included one measurement (the earliest available) per patient. 
Society (ERS/ATS) guidelines [23]. Earlier measurements were performed according to national recommendations.

\section{Statistical analysis}

We compared basic characteristics (sex, age and country) of included and excluded (no available growth measurements) patients using Chi-squared tests. We compared height and BMI z-scores of the overall study population to national and WHO reference values, using mixed linear regression models with a fixed intercept (difference to reference population), and random intercept to account for repeated measurements of the same patient. In addition, to identify the determinants of height and BMI, we included fixed effects for sex, age group, country and diagnostic certainty in these models. We tested for height and BMI differences between patient groups by performing likelihood ratio (LR) tests. We coded categorical predictor variables included in regression models, using weighted effect coding. Estimated parameters for a given category thus represent mean z-score differences from the population mean $[24,25]$. We performed sensitivity analyses in the subgroup of patients with a definite PCD diagnosis [20]. To test the robustness of our findings, we performed sensitivity analyses that included one measurement each (height and BMI), per patient.

We then repeated our analyses separately for height in children (age <20) and adults (age $\geqslant 20$ ), to investigate whether the disease delayed growth only, or also influenced final adult height. For BMI, we

TABLE 3 Height of primary ciliary dyskinesia (PCD) patients of the iPCD Cohort compared to national height references by characteristics of the study population

\begin{tabular}{|c|c|c|c|}
\hline Characteristics & Subjects $\#$ & z-score & p-value I $^{-1}$ \\
\hline Sex & & & 0.072 \\
\hline Male & 774 & $-0.22(-0.30$ to -0.13$)$ & \\
\hline Female & 827 & $-0.32(-0.40$ to -0.24$)$ & \\
\hline Age group years ${ }^{+}$ & & & $<0.001$ \\
\hline $0-9$ & 683 & $-0.27(-0.33$ to -0.21$)$ & \\
\hline $10-19$ & 683 & $-0.31(-0.37$ to -0.25$)$ & \\
\hline $20-29$ & 199 & $-0.22(-0.29$ to -0.14$)$ & \\
\hline $30-39$ & 119 & $-0.20(-0.29$ to -0.11$)$ & \\
\hline $40-49$ & 89 & $-0.22(-0.32$ to -0.12$)$ & \\
\hline$>50$ & 79 & $-0.23(-0.35$ to -0.11$)$ & \\
\hline Country ${ }^{\S}$ & & & $<0.001$ \\
\hline Australia & 55 & $-0.31(-0.60$ to -0.01$)$ & \\
\hline Belgium & 79 & $-0.66(-0.92$ to -0.40$)$ & \\
\hline Cyprus & 30 & $-0.14(-0.53$ to 0.26$)$ & \\
\hline Denmark & 91 & $-0.36(-0.59$ to -0.14$)$ & \\
\hline France & 119 & $0.18(-0.03$ to 0.39$)$ & \\
\hline Germany & 94 & -0.76 (-0.99 to -0.53$)$ & \\
\hline Israel & 142 & $-0.50(-0.70$ to -0.31$)$ & \\
\hline Italy & 38 & $-0.41(-0.77$ to -0.06$)$ & \\
\hline Netherlands & 66 & $-0.18(-0.45$ to 0.09$)$ & \\
\hline Norway & 22 & $-0.64(-1.10$ to -0.17$)$ & \\
\hline Poland & 97 & $-0.22(-0.46$ to 0.02$)$ & \\
\hline Switzerland & 47 & $-0.01(-0.32$ to 0.29$)$ & \\
\hline Turkey & 30 & $-1.09(-1.51$ to -0.66$)$ & \\
\hline UK & 277 & $-0.15(-0.29$ to -0.01$)$ & \\
\hline USA/Canada & 414 & $-0.16(-0.30$ to -0.05$)$ & \\
\hline Diagnostic certainty & & & 0.897 \\
\hline Definite PCD diagnosis $f$ & 1054 & $-0.28(-0.35$ to -0.21$)$ & \\
\hline Probable PCD diagnosis $\# \#$ & 254 & $-0.27(-0.42$ to -0.12$)$ & \\
\hline Clinical diagnosis only & 293 & $-0.24(-0.37$ to -0.10$)$ & \\
\hline
\end{tabular}

Data is presented as $\mathrm{n}$ or mean $(95 \% \mathrm{Cl})$ unless otherwise stated. Mean z-scores and $95 \% \mathrm{Cl}$ for each group were derived after adjusting for the remaining characteristics. \#: total number of subjects (N) was 1601. П: likelihood ratio test $p$-value indicating whether the characteristic explains differences in height within the study population. ${ }^{+}$: age at measurement (numbers represent the unique number of patients in each group, however, patients can be included in more than one group). §: patients from Serbia were excluded from this analysis because no national references were available. ${ }^{f}$ : defined as hallmark PCD electron microscopy findings and/or biallelic gene mutation, identified based on the ERS PCD Diagnostics Task Force guidelines. \#\# : abnormal light or high frequency video microscopy finding and/or low nasal NO value. 

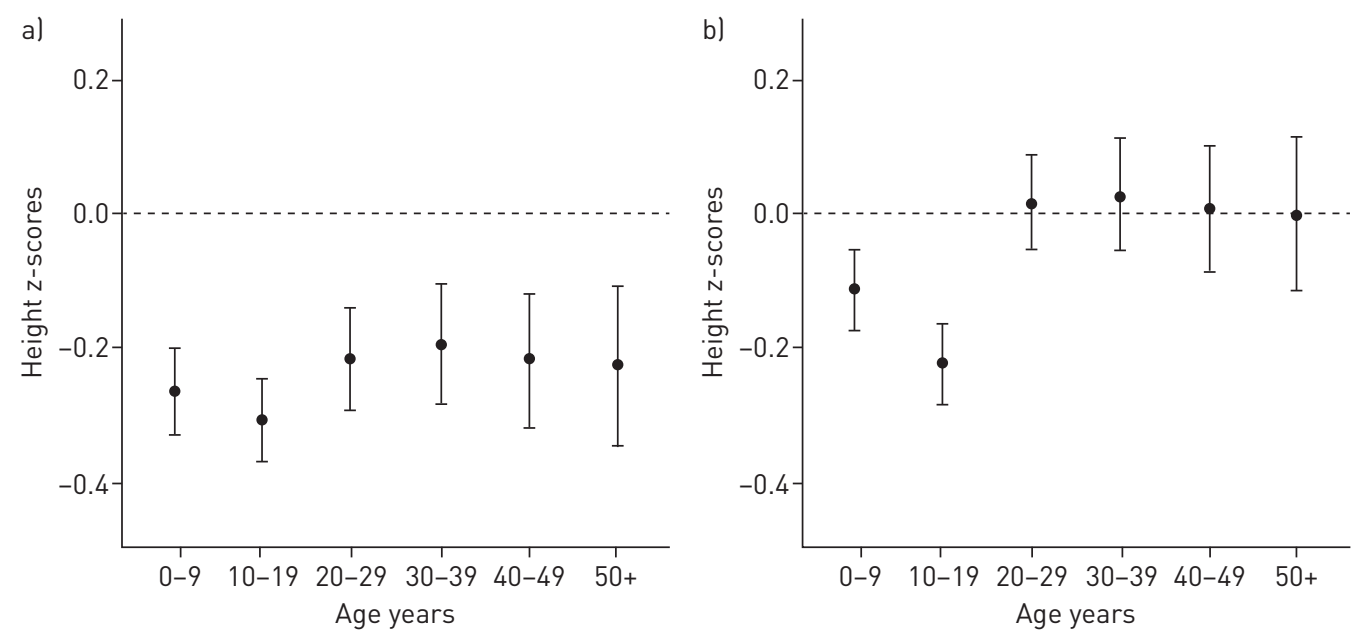

FIGURE 1 Height in primary ciliary dyskinesia (PCD) patients by age group compared to national references (a) and World Health Organization (WHO) references (b). Height is presented as mean z-score $(95 \% \mathrm{Cl})$ after adjusting for sex, country and level of diagnostic certainty.

repeated our analysis in children only, because BMI $z$-score references for $\geqslant 20$-year-olds do not presently exist. We included age at diagnosis in the multivariable linear regression models for children, to test whether age at diagnosis influenced growth and nutrition.

Finally, we assessed the association between FEV1 and FVC, and height and BMI in separate multivariable linear regression models in patients with information on lung function. The FEV1 and FVC were included as a linear term after visual inspection of scatter plots. We used the statistical programs STATA 14.1 and $\mathrm{R}$ 3.2.3 for all analyses.

\section{Results}

\section{Population characteristics}

Twenty centres delivered data from 2675 patients at the time of analysis, and 18 of the datasets from 16 countries (2131 patients) contained information on growth (supplementary figure S1). The information necessary to calculate $\mathrm{z}$-scores (height, weight, date of measurement and sex) was available for 1609 patients (6402 measurements). Analyses of organ laterality defects and lung function included 1054 and 959 patients, respectively.

Included patients were slightly older than excluded patients because of insufficient growth data (supplementary table S2, $\mathrm{p}<0.001)$, and differed by country of residence $(\mathrm{p}<0.001)$. Sex was almost evenly distributed, and more than half of all patients (58\%) came from European centres. Table 1 summarises the characteristics of the study population. Using the recent ERS PCD Diagnostics Task Force guidelines [20], $66 \%$ of patients were defined as having definite PCD, $16 \%$ as having probable PCD and $18 \%$ as having only a clinical diagnosis. Most of the patients were diagnosed during childhood ( $50 \%$ at $0-9$ years, $28 \%$ at 10-19 years).

\section{Height and BMI in PCD patients compared to national and international reference values}

The PCD patients were shorter, as compared to both national and international references (table 2). Reduced height was observed consistently across gender and age groups, and 146 patients (9\%) had a short stature (table 3, figure 1). Results differed between countries, but height was typically lower than respective national references (table 3, figure 2). Only patients living in Cyprus, France and Switzerland had normal height z-scores. The small difference in height between males and females did not quite reach statistical significance (LR p=0.072). We found no difference in height between levels of diagnostic certainty, compared to both national and WHO references (LR $\mathrm{p}=0.897$ and $\mathrm{p}=0.531$, respectively; table 3 and supplementary table S3). Height deficit differed between the age groups, with the larger deficit in children (LR p $<0.001$, table 3).

Overall, the average BMI of PCD patients was slightly above normal, as compared to national and international references (table 2). In at least one measurement during the study period, 105 patients $(6 \%)$ were underweight, whereas 405 (25\%) were overweight. Children $\leqslant 9$ years of age showed lower BMI than normal, compared to national references (supplementary table S4, figure 3). Results for BMI differed by 


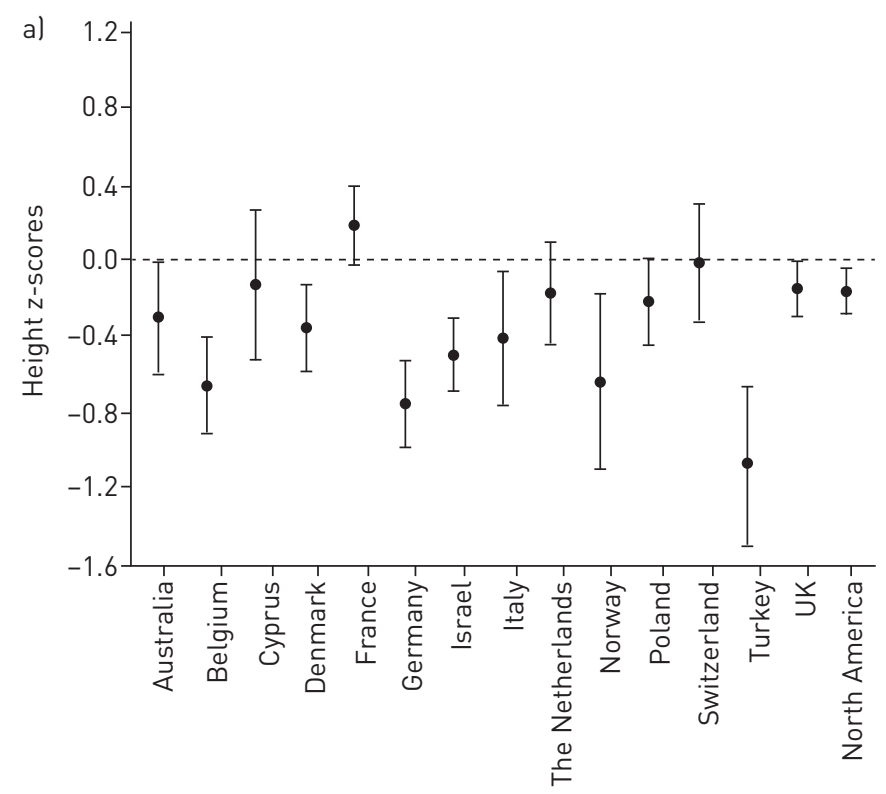

Country of residence

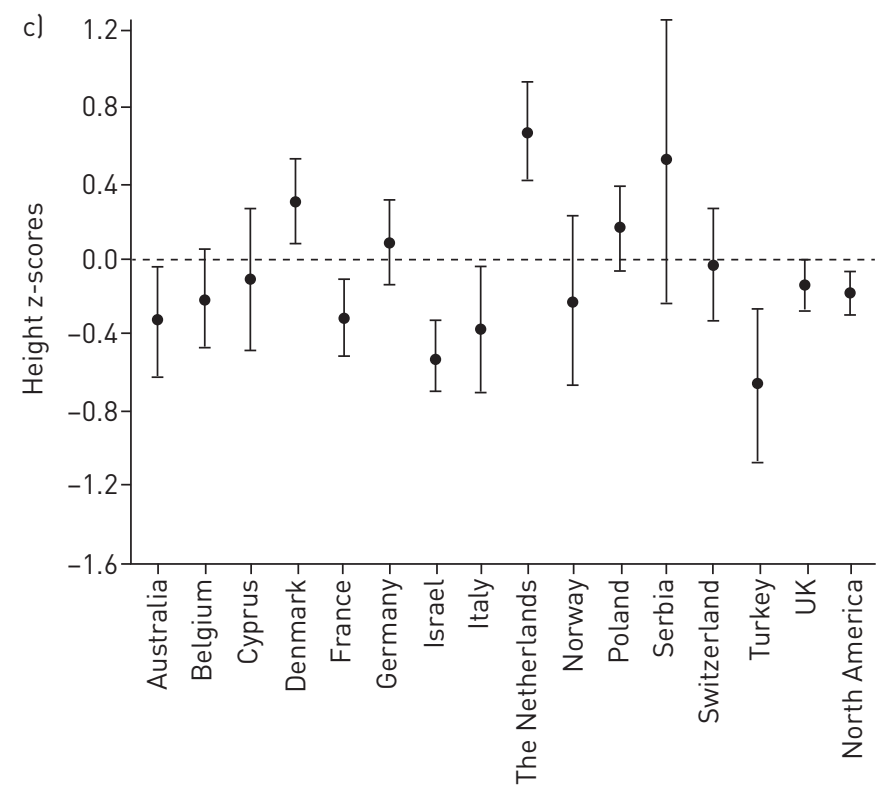

Country of residence

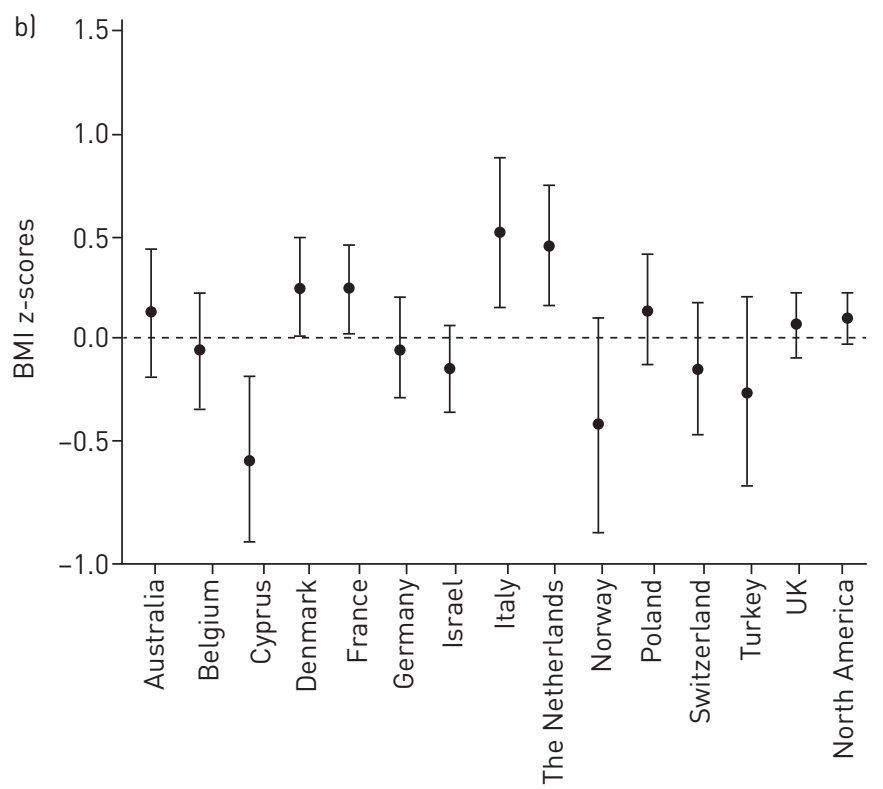

Country of residence

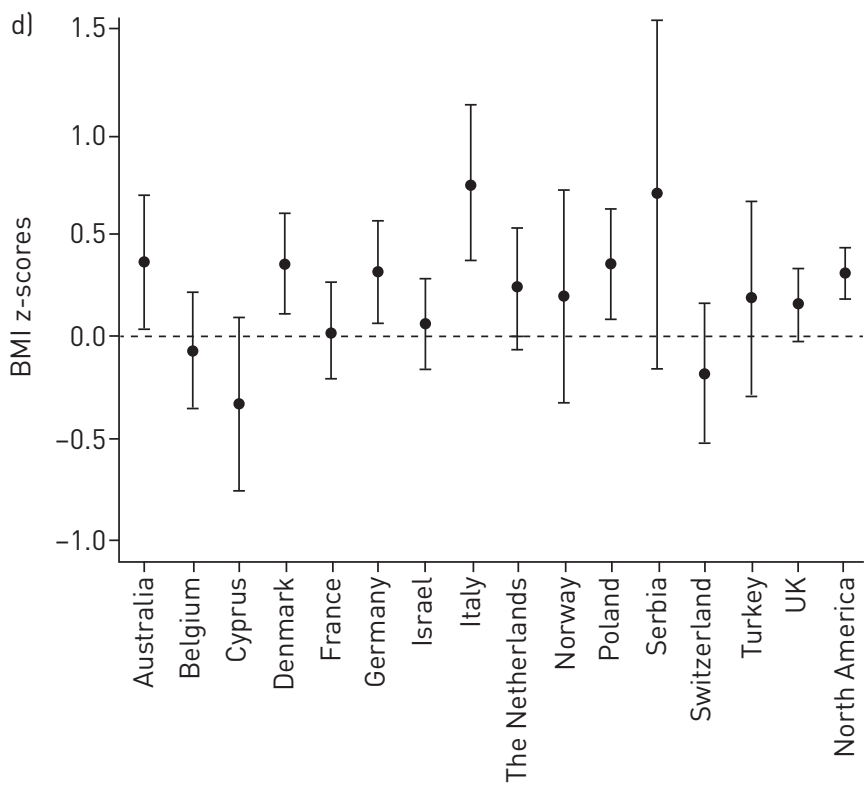

Country of residence

FIGURE 2 Height and body mass index (BMI) in primary ciliary dyskinesia (PCD) patients by country compared to national references (a) and (b) and World Health Organization (WHO) references (c) and (d). Height and BMI are presented as mean z-score (95\% CI) after adjusting for sex, age group and level of diagnostic certainty. Patients from Serbia were included only in the analysis using the WHO references because no national growth references were available.

country and by age group, with increasing z-scores in older participants (LR $\mathrm{p}<0.001$ ). In most countries, BMI was higher than national references; in a few, it was similar (supplementary table S4, figure 2). Only patients from Cyprus showed reduced BMI. The BMI was higher in males than females (LR p=0.019, supplementary table S4), but did not differ across diagnostic groups (supplementary table S4). Results were similar, when compared to the WHO references, with slightly higher z-scores in all countries, except France and the Netherlands (supplementary table S5).

Organ laterality did not predict height or BMI (supplementary figure S2, LR p >0.698). Sensitivity analyses that included only patients with a definite PCD diagnosis (1054 patients) and those that included only one measurement per patient (1601 patients) returned results for height and BMI that were similar to the original model (table 2 and supplementary tables S6-S9). 

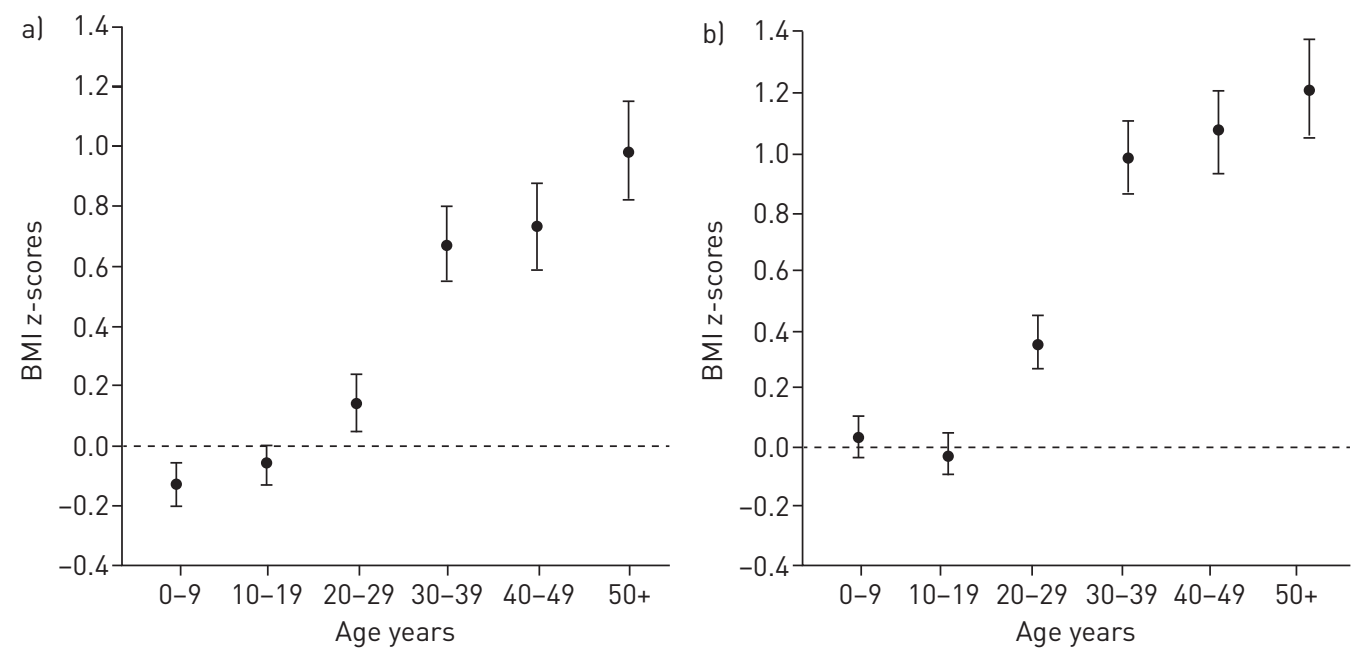

FIGURE 3 Body mass index (BMI) in primary ciliary dyskinesia (PCD) patients by age group compared to national references (a) and World Health Organization (WHO) references (b). BMI is presented as mean $\mathrm{z}$-score $(95 \% \mathrm{Cl})$ after adjusting for sex, country and level of diagnostic certainty.

Growth in children and association with age at diagnosis

Analyses of the 1226 paediatric patients confirmed reduced height in children with PCD (table 2 and supplementary table S10). The deficit differed between age groups ( $L R \mathrm{p}=0.008$, supplementary table S6). Patients diagnosed later, aged $\geqslant 15$ years, had a larger height deficit ( $L R \mathrm{p}=0.026$, supplementary table S10). The final height of the 439 adult patients was reduced (table 2 and supplementary table S7) in both sexes and all diagnostic groups (supplementary table S11), and results differed by country (LR p<0.001).

Overall, the BMI in children was similar to that in national references (tables 2 and 4), but increased with age (LR p $<0.001$ ); pre-schoolers ( $\leqslant 4$ years) had lower BMI z-scores (table 4$)$. Age at diagnosis was linearly associated with BMI $z$-score (LR $p<0.001$ ); children diagnosed earlier had a higher BMI at time of measurement than children diagnosed later in life (table 4). Children diagnosed at age $\leqslant 4$ years had higher BMI $z$-scores than normal, whereas those of children diagnosed at age $\geqslant 15$ years were lower.

\section{Association with lung function}

In 959 patients with available lung function data (median age 16 years, interquartile range 19), we found a positive association between height and BMI, and FEVland FVC measured at the same time (figure 4). Patients with a higher FEV1 z-score were taller ( $z$-score increase per additional FEV1 z-score 0.05, p<0.001; 95\% CI 0.04-0.07) and had a higher BMI z-score (z-score increase per additional FEV1 z-score 0.17, $\mathrm{p}<0.001 ; 95 \%$ CI $0.15-0.19)$. Similarly, patients with a higher FVC z-score had a higher height z-score ( $z$-score increase per additional FVC $z$-score $0.03, p<0.001 ; 95 \%$ CI $0.02-0.05$ ) and had a higher BMI $\mathrm{z}$-score (z-score increase per additional FEV1 z-score 0.17, p<0.001; 95\% CI 0.09-0.24).

\section{Discussion}

Summary of results

The height $z$-scores of PCD patients were lower than both international and national growth references. All of the multinational age groups, including fully mature adults, were shorter than the reference population. The BMI was slightly higher in the overall cohort, but was lower than normal in young children. These results were consistent across all diagnostic and organ laterality subgroups. Diagnosis at a later age was associated with lower height and lower BMI in children, and lung function (FEV1 and FVC) was positively associated with both height and BMI in males and females, and all age groups.

\section{Strengths}

The iPCD dataset represents most known PCD patients currently being followed in developed countries. Our study group is thus an order of magnitude larger than that of any previous study that has examined growth and nutrition in patients with PCD and their association with lung function. This approach has for the first time allowed the study of differences in height and BMI of PCD patients between age groups, countries and levels of diagnostic certainty.

The PCD diagnosis has changed over time and varies between countries. We therefore strengthened our analysis with sensitivity tests that included only patients with a definite PCD diagnosis, based on the 
TABLE 4 Body mass index (BMI) of paediatric patients ( $<20$ years) of the iPCD Cohort compared to national BMI references by characteristics of the study population

\begin{tabular}{|c|c|c|c|}
\hline Characteristics & Subjects ${ }^{\#}$ & z-score & $p$-value $e^{\pi}$ \\
\hline Sex & & & 0.605 \\
\hline Male & 610 & $0.04(-0.06$ to 0.13$)$ & \\
\hline Female & 574 & $-0.0002(-0.10$ to 0.10$)$ & \\
\hline Age group years $^{+}$ & & & $<0.001$ \\
\hline $0-4$ & 226 & $-0.24(-0.35$ to -0.13$)$ & \\
\hline $5-9$ & 504 & $-0.01(-0.09$ to 0.07$)$ & \\
\hline $10-14$ & 475 & $0.05(-0.03$ to 0.13$)$ & \\
\hline $15-19$ & 322 & $0.22(0.13$ to 0.31$)$ & \\
\hline Country & & & 0.004 \\
\hline Australia & 44 & $-0.07(-0.42$ to 0.28$)$ & \\
\hline Belgium & 38 & $-0.33(-0.73$ to 0.07$)$ & \\
\hline Cyprus & 17 & $-0.44(-0.98$ to 0.10$)$ & \\
\hline Denmark & 74 & $-0.01(-0.27$ to 0.25$)$ & \\
\hline France & 118 & $0.09(-0.13$ to 0.31$)$ & \\
\hline Germany & 82 & $-0.22(-0.48$ to 0.05$)$ & \\
\hline Israel & 100 & $-0.05(-0.30$ to 0.20$)$ & \\
\hline Italy & 33 & $0.41(0.02$ to 0.80$)$ & \\
\hline The Netherlands & 50 & $0.23(-0.10$ to 0.56$)$ & \\
\hline Norway & 19 & $-0.62(-1.14$ to -0.10$)$ & \\
\hline Poland & 82 & $0.02(-0.26$ to 0.30$)$ & \\
\hline Switzerland & 32 & $-0.10(-0.49$ to 0.29$)$ & \\
\hline Turkey & 30 & $-0.51(-0.97$ to -0.05$)$ & \\
\hline UK & 182 & $0.03(-0.15$ to 0.21$)$ & \\
\hline USA/Canada & 283 & $0.21(0.05$ to 0.36$)$ & \\
\hline Diagnostic certainty & & & 0.829 \\
\hline Definite PCD diagnosis $f$ & 767 & $0.004(-0.08$ to 0.09$)$ & \\
\hline Probable PCD diagnosis ${ }^{\# \#}$ & 197 & $0.03(-0.16$ to 0.21$)$ & \\
\hline Clinical diagnosis only & 220 & $0.07(-0.11$ to 0.24$)$ & \\
\hline Age at diagnosis years & & & $<0.001$ \\
\hline $0-4$ & 353 & 0.24 (0.11 to 0.38$)$ & \\
\hline $5-9$ & 416 & $0.04(-0.08$ to 0.16$)$ & \\
\hline $10-14$ & 274 & $-0.07(-0.22$ to 0.08$)$ & \\
\hline $15-19$ & 141 & $-0.43(-0.64$ to -0.22$)$ & \\
\hline
\end{tabular}

Data are presented as $\mathrm{n}$ or mean $(95 \% \mathrm{Cl})$ unless otherwise stated. Mean z-scores and $95 \% \mathrm{Cl}$ for each group were derived after adjusting for the remaining characteristics. \#: the total number of subjects (N) was 1184. ": likelihood ratio test $p$-value indicating whether the characteristic explains differences in body mass index (BMI) within the study population. ${ }^{+}$: age at measurement (numbers represent the unique number of patients in each group, however, patients can be included in more than one group). ${ }^{\S}$ : patients from Serbia were excluded from this analysis because no national references were available. ${ }^{f}$ : defined as hallmark primary ciliary dyskinesia (PCD) electron microscopy findings and/or biallelic gene mutation, identified based on the ERS PCD Diagnostics Task Force guidelines. \#\# : abnormal light or high frequency video microscopy finding and/or low nasal NO value.

recent diagnostic guidelines from the ERS PCD Diagnostics Task Force [20]. The results were further strengthened by our use of both international and national reference values, rather than having relied solely on WHO Child Growth Standards. Paediatric associations often prefer national references, which allow growth comparison of sick children with healthy peers from the same country [26].

\section{Limitations}

The iPCD Cohort includes patients who are primarily from centres that have more advanced diagnostic capabilities; patients in some countries are under-represented. Growth patterns might have been shifted as well in some countries in which national references have not been updated.

We compensated for the lack of references for adults, as other studies have done, by calculating the BMI $\mathrm{z}$-scores for adult patients, based on the reference values for 19-year-olds [27]. This is not fully satisfactory though, because as the Swiss Health Survey makes clear, BMI increases with age [28]. We therefore think our BMI results are primarily valid for children. For adults, we would need a comparison, currently beyond the scope of this study, between PCD patients and appropriate adult control groups. 

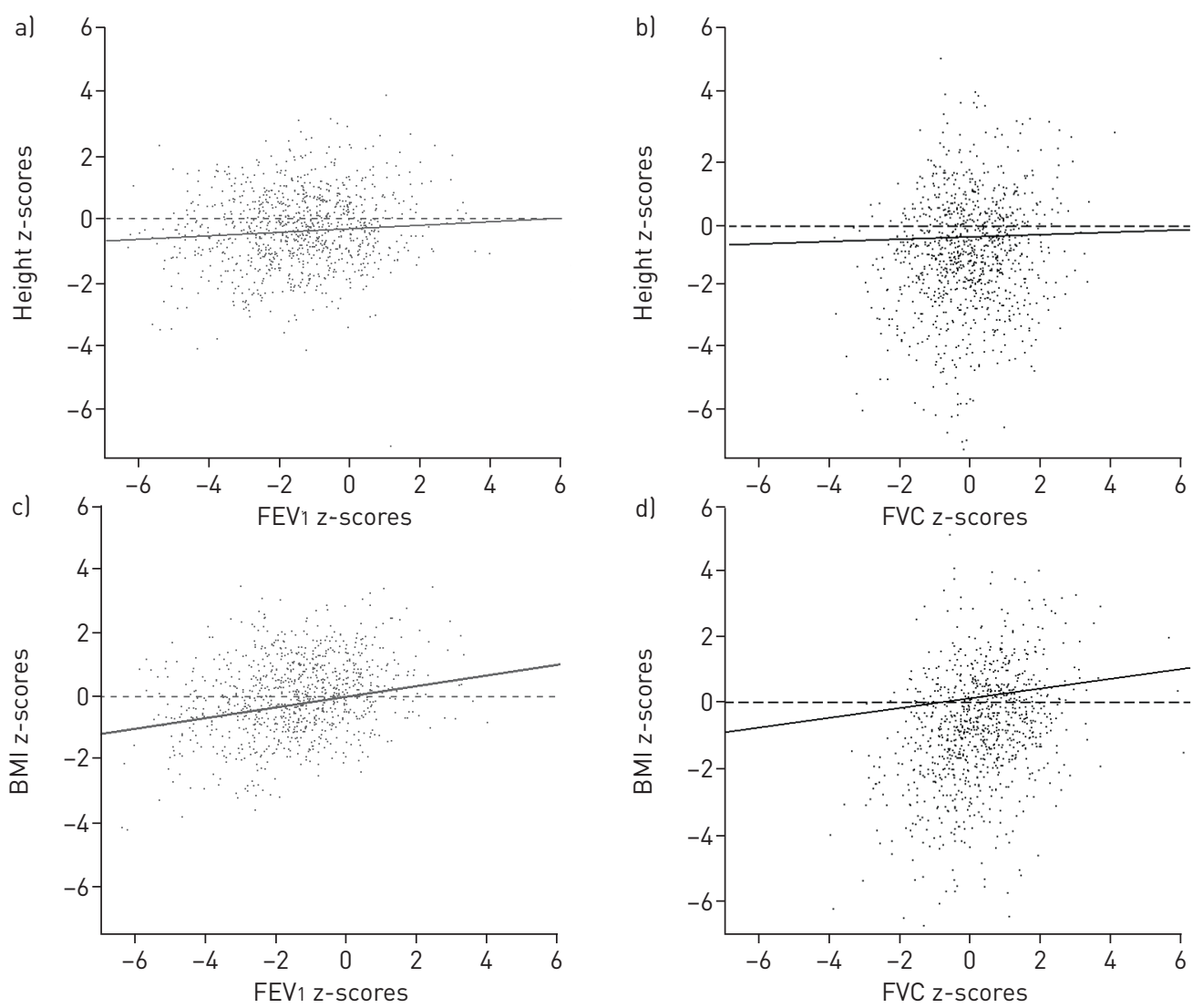

FIGURE 4 Association of height and body mass index (BMI) with forced expiratory volume in $1 \mathrm{~s}$ (FEV 1 ) (a) and (c) and forced vital capacity (FVC) (b) and (d) in primary ciliary dyskinesia (PCD) patients, using national references. Height and BMI are presented as z-scores after adjusting for sex, country and level of diagnostic certainty. FEV 1 and FVC are presented as z-scores, calculated using the Global Lung Function Initiative references. One measurement (the first available) per patient is included. The z-score increase per additional FEV1 z-score was: 1) in height: $0.05, \mathrm{p}<0.001,95 \% \mathrm{Cl} 0.04-0.07$; and 2) in $\mathrm{BMI}: 0.17, \mathrm{p}<0.001,95 \% \mathrm{Cl} 0.15-$ 0.19 . The $z$-score increase per additional FVC z-score was: 1 ) in height: $0.03, p<0.001,95 \% \mathrm{Cl} 0.02-0.05$; and 2) in BMI: 0.17, p<0.001, 95\% $\mathrm{Cl} 0.09-0.24$.

Information on ethnicity was not recorded for all patients; therefore, height and BMI z-scores were calculated using national references of countries of residence. For patients of unknown ethnicity, we calculated FEV1 and FVC $\mathrm{z}$-scores using the other/mixed GLI reference values. We assume that this affected our results only marginally, particularly because patients with possible South Asian origin, who are known to have different lung function reference values, comprised less than $5 \%$ of our study population.

\section{Comparison with other studies}

Results of previous studies of growth and PCD vary considerably (supplementary table S12). In a Belgian comparison of 168 PCD patients with national references, the PCD patients were shorter ( $\mathrm{z}$-score -0.53 , 95\% CI -0.85 to -0.22 ), but had normal BMI values [13]. In six North American centres, most of the 118 children fell within normal parameters, but children with CCDC39 and CCDC40 mutations showed reduced height, BMI and FEV1, compared to those with different mutations [16]. A comparison of the height and BMI of 158 paediatric patients at three centres in Italy, Denmark and the UK, which used UK growth references, concluded that height and BMI were normal [17]. Another Czech comparison of PCD patients to national references found that children aged 7-13 years were shorter, even though the BMI in all age groups was normal. Their height $\mathrm{z}$-scores declined during childhood until age 9 years, but height difference did not depend on age at diagnosis [14]. However, each of these comparisons with respective $\mathrm{UK}$ and Czech national references showed minimal differences and lacked power.

For other respiratory diseases, associations between growth and nutrition, and lung function have been studied extensively. Taking the example of bronchopulmonary dysplasia, the nutritional status of 2-year-olds predicted lung function in later childhood [6]. Similarly, CF patients with better growth and 
nutrition at 3 years of age also had better lung function at 6 years [7]. Furthermore, in a comparison of PCD and CF patients in Israel, BMI was lower in 34 PCD patients than it was in 130 CF patients (with or without pancreatic insufficiency), and BMI and FEV1 tended towards correlation $(\mathrm{r}=0.44, \mathrm{p}=0.06)$ [15].

\section{Interpretation of results}

What do these findings all mean? It is not possible to derive the direction of the association between growth and lung function from cross-sectional data. Yet, it certainly is possible and probable that poorer lung function, resulting in increased respiratory effort and higher resting energy expenditure, can affect growth in PCD patients. Impaired nutrition, caused, for example, by reduced appetite owing to swallowed sputum or repeated infections, could further impair lung development [29]. This is supported by evidence from CF studies, which indicate that nutritional support helps to reduce mortality [30, 31].

Our data suggest more strongly than any other obtained to date that growth in PCD patients starts to diverge from the norm at an early age. This could imply a genetic cause. Prevalence of PCD is higher in consanguineous populations [32], and inbreeding reportedly contributes to reduced height [33]. Primary (nonmotile) cilia play a role in mechanical signalling in bone cells, but a possible role in skeletal growth has not been tested [34]. A recent study has reported mild scoliosis in 23\% of 198 UK PCD patients, which could have an additional effect that reduces height [35]. Height and BMI were lower in children with CCDC39 and CCDC40 mutations [16], which cause specific ultrastructural defects (absence of inner dynein arms and microtubular disorganisation), and affect up to $12 \%$ of all PCD cases [36, 37]. We had genetic data for only a few patients and could not pursue this any further. However, based on their low prevalence, these mutations alone could not explain our findings.

Chronic and recurrent infections, which are common in PCD, could negatively affect the periods of rapid growth in childhood, when the nutrients needed for growth might be used for immune defence [38, 39]. Alternatively, oral or inhaled corticosteroids (ICS) might play a role. An earlier European survey of PCD centres reported routine use of ICS in 15\% of all centres, particularly in Northern Europe [40]. The PCD patients might also have been misclassified as severe asthmatics and treated with systemic corticosteroids or high-dose ICS prior to their PCD diagnosis. The iPCD Cohort has data on corticosteroid use for only a few patients, so we were unable to test this hypothesis.

\section{Conclusions}

Future research should collect longitudinal data, ideally from birth through adulthood, to evaluate changes in height, BMI and lung function over time, and investigate the direction of their associations using appropriate statistical approaches [41]. This would also allow assessment of the influence of different treatment strategies and recurrent infections on growth and the course of lung function. From a clinical perspective, our findings suggest that early diagnosis, followed by multidisciplinary management could delay disease progression and improve growth. Monitoring height and BMI, and if necessary, early nutritional interventions should become part of routine care in PCD clinics, to maximise lung growth and delay disease progression.

\section{Acknowledgements}

We want to thank all patients in the PCD cohort and their families, and we are grateful to the PCD patient organisations that closely collaborated with us. We thank all the researchers in the participating centres who helped collect and enter data, and worked closely with us throughout building the iPCD Cohort. We thank Zorica Zivkovic (Children's Hospital for Lung Diseases and TB, Medical Centre "Dr Dragisa Misovic", Belgrade, Serbia) for contributing patients from her centre. We appreciate the work of Edwige Collaud and Anna Bettina Meier (Institute of Social and Preventive Medicine, University of Bern, Switzerland) who helped us with the height and BMI z-score calculations for some countries. We also thank Kali Tal and Christopher Ritter (Institute of Social and Preventive Medicine, University of Bern, Switzerland) for their editorial suggestions.

Author Contributions: C.E. Kuehni and M. Goutaki developed the concept and designed the study. M. Goutaki and F.S. Halbeisen cleaned and standardised the data. M. Goutaki performed the statistical analyses under the guidance of B.D. Spycher. All other authors participated in discussions for the development of the study and contributed data. C.E. Kuehni, M. Goutaki, B.D. Spycher, F.S. Halbeisen and J.S. Lucas drafted the manuscript. All authors contributed to iterations and approved the final version. C.E. Kuehni and M. Goutaki take final responsibility for the contents.

\section{References}

1 Chang KC, Miklich DR, Barwise G, et al. Linear growth of chronic asthmatic children: the effects of the disease and various forms of steroid therapy. Clin Allergy 1982; 12: 369-378.

2 Shohat M, Shohat T, Kedem R, et al. Childhood asthma and growth outcome. Arch Dis Child 1987; 62: 63-65.

3 Zeitlin SR, Bond S, Wootton S, et al. Increased resting energy expenditure in childhood asthma: does this contribute towards growth failure? Arch Dis Child 1992; 67: 1366-1369.

4 Balfour-Lynn L. Growth and childhood asthma. Arch Dis Child 1986; 61: 1049-1055.

5 Zhang L, Prietsch SO, Ducharme FM. Inhaled corticosteroids in children with persistent asthma: effects on growth. Cochrane Database Syst Rev 2014; CD009471. 
6 Bott L, Beghin L, Devos P, et al. Nutritional status at 2 years in former infants with bronchopulmonary dysplasia influences nutrition and pulmonary outcomes during childhood. Pediatr Res 2006; 60: 340-344.

$7 \quad$ Konstan MW, Butler SM, Wohl ME, et al. Growth and nutritional indexes in early life predict pulmonary function in cystic fibrosis. J Pediatr 2003; 142: 624-630.

8 Liou TG, Adler FR, Fitzsimmons SC, et al. Predictive 5-year survivorship model of cystic fibrosis. Am J Epidemiol 2001; 153: 345-352.

9 van den Bemt L, Schermer T, Smeele I, et al. Monitoring of patients with COPD: a review of current guidelines' recommendations. Respir Med 2008; 102: 633-641.

10 de Onis M, Wijnhoven TM, Onyango AW. Worldwide practices in child growth monitoring. J Pediatr 2004; 144: 461-465.

11 Kuehni CE, Frischer T, Strippoli MP, et al. Factors influencing age at diagnosis of primary ciliary dyskinesia in European children. Eur Respir J 2010; 36: 1248-1258.

12 Lucas JS, Walker WT, Kuehni CE, et al. Primary ciliary dyskinesia. In: Courdier J-F, ed. Orphan Lung Diseases (ERS Monograph). Sheffield, European Respiratory Society, 2011; pp. 201-217.

13 Boon M, Smits A, Cuppens $\mathrm{H}$, et al. Primary ciliary dyskinesia: critical evaluation of clinical symptoms and diagnosis in patients with normal and abnormal ultrastructure. Orphanet J Rare Dis 2014; 9: 11.

14 Svobodová T, Djakow J, Zemkova D, et al. Impaired growth during childhood in patients with primary ciliary dyskinesia. Int J Endocrinol 2013; 2013: 731423.

15 Cohen-Cymberknoh M, Simanovsky N, Hiller N, et al. Differences in disease expression between primary ciliary dyskinesia and cystic fibrosis with and without pancreatic insufficiency. Chest 2014; 145: 738-744.

16 Davis SD, Ferkol TW, Rosenfeld M, et al. Clinical features of childhood primary ciliary dyskinesia by genotype and ultrastructural phenotype. Am J Respir Crit Care Med 2015; 191: 316-324.

17 Maglione M, Bush A, Nielsen KG, et al. Multicenter analysis of body mass index, lung function, and sputum microbiology in primary ciliary dyskinesia. Pediatr Pulmonol 2014; 49: 1243-1250.

18 Goutaki M, Maurer E, Halbeisen FS, et al. The international primary ciliary dyskinesia cohort (iPCD Cohort): methods and first results. Eur Respir J 2017; 49: 1601181.

19 Lucas JS, Paff T, Goggin P, et al. Diagnostic methods in primary ciliary dyskinesia. Paediatr Respir Rev 2016; 18: $8-17$.

20 Lucas JS, Barbato A, Collins SA, et al. European Respiratory Society guidelines for the diagnosis of primary ciliary dyskinesia. Eur Respir J 2017; 49: pii 1601090.

21 Group WMGRS. WHO child growth standards: length/height-for-age, weight-for-age, weight-for-length, weight-for-height and body mass index-for-age-methods and development. Geneva, World Health Organization, 2006

22 Quanjer PH, Stanojevic S, Cole TJ, et al. Multi-ethnic reference values for spirometry for the 3-95-yr age range: the global lung function 2012 equations. Eur Respir J 2012; 40: 1324-1343.

23 Miller MR, Hankinson J, Brusasco V, et al. Standardisation of spirometry. Eur Respir J 2005; 26: 319-338

24 Davis M. Contrast coding in multiple regression analysis: strengths, weaknesses, and utility of popular coding structures. J Data Sci 2010; 8: 61-73.

25 Wendorf C. Primer on multiple regression coding: common forms and the additional case of repeated contrasts. Understanding Stat 2004; 3: 47-57.

26 Bonthuis M, van Stralen KJ, Verrina E, et al. Use of national and international growth charts for studying height in European children: development of up-to-date European height-for-age charts. PLoS One 2012; 7: e42506.

27 Must A, Anderson SE. Body mass index in children and adolescents: considerations for population-based applications. Int J Obes (Lond) 2006; 30: 590-594.

28 Eichholzer M, Bovey F, Jordan P, et al. Daten rund um das Körpergewicht: Schweizerische Gesundheitsbefragung 2007 [Body weight related data: results of the 2007 Swiss Health Survey]. Praxis (Bern 1994) 2010; 99: 895-906.

29 Korten I, Usemann J, Latzin P. "Lung sparing growth": is the lung not affected by malnutrition? Eur Respir J 2017; 49: 1700295 .

30 Dodge JA, Lewis PA, Stanton M, et al. Cystic fibrosis mortality and survival in the UK: 1947-2003. Eur Respir J 2007; 29: 522-526.

31 Stephenson AL, Mannik LA, Walsh S, et al. Longitudinal trends in nutritional status and the relation between lung function and BMI in cystic fibrosis: a population-based cohort study. Am J Clin Nutr 2013; 97: 872-877.

32 O'Callaghan C, Chetcuti P, Moya E. High prevalence of primary ciliary dyskinesia in a British Asian population. Arch Dis Child 2010; 95: 51-52.

33 McQuillan R, Eklund N, Pirastu N, et al. Evidence of inbreeding depression on human height. PLoS Genet 2012; 8: e1002655.

34 Lee KL, Guevarra MD, Nguyen AM, et al. The primary cilium functions as a mechanical and calcium signaling nexus. Cilia 2015; 4: 7.

35 Schlösser TPC, Semple T, Carr SB, et al. Scoliosis convexity and organ anatomy are related. Eur Spine J 2017; 26: 1595-1599.

36 Papon JF, Coste A, Roudot-Thoraval F, et al. A 20-year experience of electron microscopy in the diagnosis of primary ciliary dyskinesia. Eur Respir J 2010; 35: 1057-1063.

37 Shoemark A, Dixon M, Corrin B, et al. Twenty-year review of quantitative transmission electron microscopy for the diagnosis of primary ciliary dyskinesia. J Clin Pathol 2012; 65: 267-271.

38 Pammi M, Vallejo JG, Abrams SA. Nutrition-infection interactions and impacts on human health. Boca Raton, CRC Press, 2014.

39 Stephensen CB. Burden of infection on growth failure. J Nutr 1999; 129: 534s-538s.

40 Strippoli MP, Frischer T, Barbato A, et al. ERS task force on primary ciliary dyskinesia in children. Management of primary ciliary dyskinesia in European children: recommendations and clinical practice. Eur Respir J 2012; 39: 1482-1491.

41 Galea S, Riddle M, Kaplan GA. Causal thinking and complex system approaches in epidemiology. Int J Epidemiol 2010; 39: 97-106. 